\title{
A Different Aproach of Chronic Obstructive Pulmonary Disease Severity and Plastic Medical Devices Used for Oxygenotherapy
}

\author{
DOINA ECATERINA TOFOLEAN ${ }^{1}$, GILDA POPESCU2*, IOAN ANTON ARGHIR ${ }^{3}$, MIRELA FRANDES ${ }^{4}$, \\ ARIADNA PETRONELA FILDAN ${ }^{1}$ \\ ${ }^{1}$ Ovidius University of Constanta, Faculty of Medicine, Internal Medicine Department, 124 Mamaia Blvd, 900527, Constanta, \\ Romania \\ ${ }^{2}$ Titu Maiorescu University, Faculty of Medicine, Pulmonology Department, 22 Dambovnicului Tineretului Str, 040441, \\ Bucharest, Romania \\ ${ }^{3}$ Constanta County Clinical Emergency Hospital St. Andrew, Pulmonology Department, 145 Tomis Blvd, 900591, Constanta, \\ Romania \\ ${ }^{4}$ University of Medicine and Pharmacy Victor Babes, Department of Biostatistics and Medical Informatics, 2 Eftimie Murgu \\ Sq., 300041, Timisoara, Romania
}

\begin{abstract}
A very common questionnaire, used to evaluate the health status of Chronic Obstructive Pulmonary Disease (COPD) patients, COPD assessment test (CAT), was applied to finding correlations between the items of CAT and other tests used in the same category of COPD patients. A sample of 56 male COPD patients, aged between 51 and 74 years, mean 63.86 ( \pm 5.55$)$, half of them receiving long-term oxygen therapy, using transparent, hypoallergenic plastic masks or nasal cannulas, answered to CAT mostly choosing single items, statistically significant correlated with the Hospital Anxiety and Depression Scale scores (HADs), like walking up hills and stairs $(r=0.412, p<0.01)$, doing activities at home $(r=0.329, p<0.01)$, confidence leaving my home $(r=0.409, p<0.001)$, sleep $(r=0.277, p<0.01)$, and energy $(r=0.387, p<0.01)$, but CAT item walking up hills and stairs correlated better to 6 min walking distance $(6 M W D)(r=-0.581, p<0.01)$. The most significant correlations were found between 3 items of CAT as walking up hills and stairs, limitations doing activities at home and confidence leaving home in a most powerful and depression scores and 6MWD.
\end{abstract}

Keywords: COPD assessment test CAT, 6 min walking distance, anxiety, depression, plastic masks.

Quality of life is an important objective in the management of chronic obstructive pulmonary disease (COPD) that has been highlighted as a future research need [1]. Some of the most important systemic and pulmonary manifestations of COPD can lead to dyspnoea and physical exertion that eventually aggravate symptoms, can progressively impair the functional status and the activities of daily living [2-4]. An important recommendation has been issued by the Global Initiative of Chronic Obstructive Lung Disease (GOLD) since 2011. This strategy highlights that the management and treatment of COPD should include beside the spirometric values, also the disease impact on future risk of exacerbations and progression $[2,5,6]$. Several questionnaires of the quality of life are generally used to evaluate self-reported health status in COPD patients but one of the most known questionnaires is the COPD assessment test (CAT) $[7,8]$. This questionnaire offers an alternative to other complex tools used for COPD patients such as the St. Georges Respiratory Questionnaire and the Chronic Respiratory Questionnaire [7]. With CAT help, the patient state can be easily understood because of its simple and extensive quality. Due to its importance, the GOLD strategy considered to create a new classification of COPD, measuring the risk of exacerbations [2]. There are still problems concerning the importance and the evidence in supporting the use of CAT questionnaire [8]. Some studies have shown that, when calculating the entire CAT score, the importance is distributed to all the single items from the questionnaire. Other studies are launching the hypothesis that single items can be more strongly related to differentseverity categories of the disease. As an example, phlegm and cough can be better related to respiratory related events than others. Furthermore, patients with chronic hyper-secretion are more susceptible to have exacerbations thus leading to more hospitalizations [9]. Taking into account the above mentioned facts, the objective of the study was to analyze and find correlations between the items of CAT and other tests usually used in COPD patients.

\section{Experimental part}

A transversal study was assessed among patients known with severe and very severe GOLD stages of COPD [1] during periodical routine control during the period 01.06 - 01.12. 2017. An informed consent was signed by all the patients before entering the study. The research was approved by the Pulmonology hospital from Constanta (number 2662-10052017). Because the great majority of hospitalized COPD cases are males, for statistical reasons, only male patients have been included. Exclusion criteria consisted in previous syncope, known cognitive impairment, postural orthostatic hypotension, severe cardiovascular disease, and/or transient ischemic attacks.

Several procedures were assessed. Lung function was evaluated by volumes obtained using MedGhraphics spirometer and maximal inspiratory/expiratory pressure (MIP, MEP) values were acquired using Jaeger (Germany) device. All cases of overlap asthma COPD or asthma COPDlike, mentioned by literature [10], were excluded. Patients with respiratory failure received long-term oxygen therapy through stationary concentrators and oxygen masks or cannulas. The medical devices used for oxygen delivery were made from hypoalergenic plastic, using polymer compounds that have been approved for biocompatibility,

\footnotetext{
*email: popescugilda@yahoo.com
} 
sterilization, and material physical performance. Biocompatibility of polymer compounds reffers to the absence of any adverse reaction of the human body and is demonstrated using three tests (direct contact, agar diffusion, and elution) [11].

Plastic compounds have been traditionally derived from petroleum products. More often, in the present, some of the plastic compounds are made using biobased materials, such as polymers made from renewable resources and, also, biobased additives. Biobased materials, including plasticizers, stabilizers, and lubricants, are considered more durable and environmentally friendly. Regarding the specific types of polymers, the most used polymer for medical devices was PVC, because of its many properties, including versatility, clarity, availability, kink resistance, and low price. In order to gain other enhanced properties such as resilience, chemical and abrasion resistance, there were developed PVC alloys (e.g., with polyurethane, ethylene vinyl acetate, and polyacrylonitrile) and thermoplastic elastomer (TPE) compounds, which are more flexible without containing plasticizers. [12]

Bioelectrical impedance analysis (BIA) was used to assess body composition by a body composition device, respecting the established protocol for BIA analysis [13]. Montreal Cognitive Assessment test (MoCA), used to analyse the cognitive function, evaluated the cognitive dysfunction using different domains such as memory, language, executive functions, concentration and other skills. MoCA is a 30 point test; a score below 26 points shows a mild cognitive dysfunction [14]. Functional performance was obtained through the 6 minutes walking test (6MWT), which measures the distance that a patient can quickly walk in a period of 6 minutes. It evaluates the global and integrated responses of all the systems involved during exercises, such as the pulmonary and cardiovascular systems and it assesses the sub-maximal level of functional capacity [15]. Health status was assessed with the help of the CAT questionnaire, which is commonly used in the routine practice. CAT is composed of eight questions, each item having a six point differential scale and having a maximal score of 40 points. The questions are: q1- cough, q2- phlegm, q3- chest tightness, q4- walking up hills and stairs, q5- activities doing at home, q6- confidence leaving home, q7- sleep, q8- energy. A higher score means a greater impact of the disease on the patient's health status [16]. HADs measure anxiety and depression in a general medical population of patients, including 14 items, seven related to anxiety symptoms and seven to depressive symptoms. The scores can be between 0 and 21 for each subscale: a score of 0-7 indicates a noncase, 8-10 a possible case, and 11 or higher a probable case, which may guide referral for psychological support [17]. Statistical analysis of all collected data, by using the SPSS v.17 software (SPSS Inc., Chicago, IL, USA) and R project packages for statistical computing, presented means and standard deviations for continuous variables with Gaussian distribution, median and interquartile range (IQR) for continuous variables without Gaussian distribution, or percentage (absolute frequency) for categorical variables. Continuous variable distributions were tested for normality using the Shapiro-Wilk's test and for equality of variances by using Levene's test. To assess the differences between groups, the Student's $t$-test (means, Gaussian populations), One-way ANOVA (means, Gaussian populations), Mann-Whitney $U$ test (medians, non-Gaussian populations), and Pearson chi-square or Fisher's exact test (proportions) were used. The correlation between studied variables was evaluated using
Spearman's rank sum correlation coefficient (nonGaussian distributed variables), its statistical significance being assessed using the $t$-distribution score test. A $P$ value less than 0.05 was considered as the threshold for statistical significance.

\section{Results and discussions}

A sample of 56 male patients with severe and very severe forms of COPD, mean aged $63.86( \pm 5.55)$ years (limits between 51 and 74 years), had a median CAT score of 26.00 (range 23.00-30.00). Almost a half of them (58.93\%) had persistent symptoms with a total CAT score between 20 and 29 points. The median forced vital capacity (FVC) was $2.52 \mathrm{~L}$ (range $2.29-2.94 \mathrm{~L}$ ), and the median FVC [\%] was decreased below $70 \%$ (64.50\%; range, $60.00 \%-68.55 \%$ ). Additionally, the median forced expiratory volume in the $1^{\text {st }}$ second (FEV1) was $0.91 \mathrm{~L}$ (range, 0.75 $1.15 \mathrm{~L}$ ), and the median FEV1 [\%] was $28.95 \%$ (range, $22.30-32.25 \%)$. More than one COPD exacerbation reported in the last 3 months were noticed among $17.90 \%$ patients. Almost a half of the patients were ex-smokers (73.20\%), and $26.80 \%$ were current smokers. Half of the patients received long-term oxygen therapy through stationary concentrators and oxygen masks or cannulas, made from transparent, hypoallergenic plastic. The patients' characteristics are presented in table 1.

Higher CAT scores were associated with poor lung function and more frequent exacerbations in the last 3 months. Anxiety and depression tended to be more severe at higher CAT scores, statistically significant differences being observed between score's categories (One-way ANOVA test, $F(1.54)=19.89, p<0.001$, and $F(1.54)=$ $15.55, p<0.001$, respectively). A severe decrease of physical activity capacity was found to be associated with higher CAT scores, differences between medium, high and very high CAT score levels being were statistically significant (One-way ANOVA test, $F(1.54)=6.19, p<0.05$ ) (table 2).

Lung function parameters as FVC, FEV1, FEV1/FVC were poorly correlated with total CAT score, $r=-0.105, p=0.440$; $r=0.237, p=0.079$; and $r=-0.252, p=0.060$, respectively. This could be explained by the diversity of factors influencing lung function such as treatment or nutrition status $[16,17]$. The MoCA score was also weakly correlated with the total CAT score $(r=-0.174, p=0.200)$. On the contrary, the HADs-anxiety statistically significant correlated with the total CAT score $(r=0.531, p<0.01)$, as well as HADs-depression $(r=0.408, p<0.01)$. The physical activity parameter 6MWT was statistically significant correlated with the total CAT score $(r=-0.407, p<0.01)$. At the same time, CRP inversely correlated with the total CAT score $(r=-0.286, p<0.05)$. The aim of the study was to identify correlations between single items of the CAT and the outcome parameters of COPD, such as, spirometry measures, physical activity, cognitive capacity, and health related status. So, most of the CAT items correlated alone significantly with the HAD depression scores, namely, as walking up hills and stairs $(r=0.412, p<0.01)$, doing activities at home $(r=0.329, p<0.01)$, confidence leaving my home $(r=0.409, p<0.001)$, item sleep $(r=0.277, p<$ $0.01)$, and energy $(r=0.387, p<0.01)$. CAT item cough poorly correlated with all the COPD measured parameters, as well as the item breathless when walking. CAT item chest tightness best correlated with FEV1 $(r=-0.314, p<$ $0.05)$, fibrinogen $(r=-0.379, p<0.01)$, and 6MWD ( $r=-$ $0.339, p<0.05$ ). CAT item walking up hills and stairs best correlated with 6MWD $(r=-0.581, p<0.01)$, HADsdepression $(r=0.412, p<0.01)$, and HADs-anxiety $(r=$ 


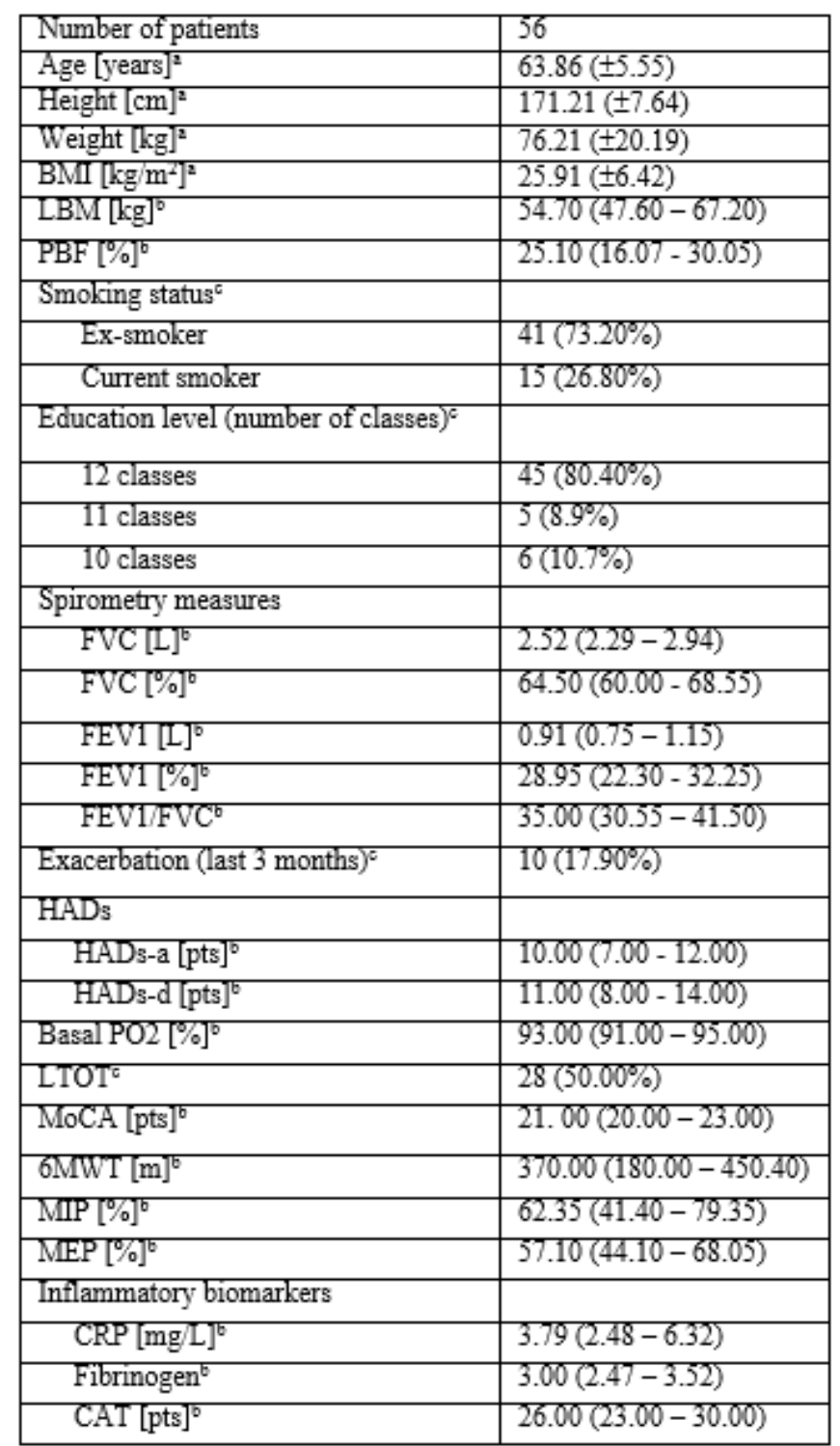

Table 1

PATIENTS BASELINE CHARACTERISTICS

${ }^{a}$ Continuous variables (with Gaussian distribution) are indicated by their mean and standard deviation.

${ }^{b}$ Continuous variables (with non-Gaussian distribution) are indicated by their median (interquartile range).

'Nominal variables are indicated by their absolute and relative frequencies.

Abbreviations: BMI - Body Mass Index; LBM - Lean Body Mass; PBF - Percent Body Fat; FVC - Forced Vital Capacity; FEV1 - Forced Expiratory Volume in the first second; HADs - Hospital Anxiety and Depression Scale; LTOT - Long-Term Oxygen Therapy;

MoCA - Montreal Cognitive Assessment; 6MWD - 6 min Walking Distance; MIP - Maximum Inspiratory Pressure; MEP - Maximum Expiratory Pressure; CRP - C-Reactive Protein; CAT - COPD Assessment Test.

\begin{tabular}{|c|c|c|c|c|}
\hline \multirow{2}{*}{ Parameter } & \multicolumn{3}{|c|}{ CAT score category } & \multirow{3}{*}{ p-value } \\
\hline & $10-19$ & $20-29$ & $30-40$ & \\
\hline Number of patients & $7(12.50 \%)$ & $33(58.93 \%)$ & $16(28.57 \%)$ & \\
\hline \multicolumn{5}{|l|}{ Lung volumes } \\
\hline FVC [L] & $2.66(0.48)$ & $2.66(0.47)$ & $2.50(0.36)$ & 0.211 \\
\hline FEV1 [L] & $0.94(0.21)$ & $1.01(0.37)$ & $0.82(0.29)$ & 0.081 \\
\hline FEV1/FVC & $35.07(4.84)$ & $37.10(11.39)$ & $32.76(10.66)$ & 0.207 \\
\hline \multicolumn{5}{|c|}{ Inflammatory biomarkers } \\
\hline Fibrinogen $[\mathrm{mg} / \mathrm{L}]$ & $2.99(0.71)$ & $2.99(0.71)$ & $3.55(3.25)$ & 0.886 \\
\hline C-reactive protein & $3.19(2.52)$ & $3.19(2.52)$ & $6.59(5.44)$ & 0.857 \\
\hline \multicolumn{5}{|c|}{ Physical activity capacity } \\
\hline $6 \mathrm{MWD}[\mathrm{m}]$ & $449.57(100.12)$ & $449.70(104.14)$ & $239.44(170.44)$ & 0.016 \\
\hline \multicolumn{5}{|l|}{ Tests } \\
\hline HADs-anxiety & $9.86(5.84)$ & $9.12(2.83)$ & $13.69(3.13)$ & $\leqslant 0.001$ \\
\hline HADs-depression & $9.71(5.91)$ & $9.30(3.14)$ & $13.38(2.70)$ & $=0.001$ \\
\hline MoCA & $21.43(2.44)$ & $21.52(2.17)$ & $20.63(2.18)$ & 0.181 \\
\hline
\end{tabular}

Table 2

COPD PARAMETERS BY DIFFERENT CATEGORIES OF THE CAT SCORE

Abbreviations: FVC - Forced Vital Capacity; FEV1 - Forced Expiratory Volume in the first second; HADs - Hospital Anxiety and Depression Scale; MoCA - Montreal Cognitive Assessment; 6MWD - 6 min Walking Distance; CRP - C-Reactive Protein; CAT - COPD Assessment Test.

$0.400, p<0.01$ ), as well as the vital capacity parameters, namely, FEV1 ( $r=-0.428, p<0.01)$, FEV1 $\%$ predicted $(r=$ $-0.394, p<0.001)$, and FEV1/FVC $(r=-0.428, p<0.01)$. CAT item have lots of energy was also correlated with HADs-anxiety $(r=0.359, p<0.01)$, HADs-depression $(r=$ $0.387, p<0.001$ ), and inversely weak but significant correlations were found with MoCA score $(r=-0.254, p<$ 0.05). CAT item limitations doing activities at home was strongly significant correlated with 6MWD $(r=-0.631, p<$ 0.01 ), HADs-depression ( $r=0.329, p<0.05)$ and HADsanxiety $(r=0.380, p<0.01)$. CAT item confident leaving my home inversely significantly correlated with 6MWD $(r$ $=-0.558, p<0.01)$, HADs-depression $(r=0.409, p<0.01)$ and HADs-anxiety $(r=0.495, p<0.01)$. Although vital capacity poorly correlated with the total CAT score, several items statistically significant correlated, such as walking up hills and stairs, and doing activities at home (table 3). 


\begin{tabular}{|c|c|c|c|c|c|c|c|c|}
\hline \multirow{2}{*}{ Parameter } & \multicolumn{8}{|c|}{ CAT questions } \\
\hline & q1 & $q^{2}$ & $q^{3}$ & $q 4$ & $q 5$ & q6 & $q^{7}$ & q8 \\
\hline \multicolumn{9}{|c|}{ Vital capacity } \\
\hline FVC [L] & 0.049 & -0.080 & -0.201 & -0.145 & -0.193 & -0.044 & 0.199 & 0.017 \\
\hline FVC [\%] & 0.006 & 0.018 & 0.080 & 0.066 & 0.024 & 0.033 & 0.144 & -0.021 \\
\hline FEV1 [L] & 0.052 & -0.026 & $-0.314^{*}$ & $-0.428 * *$ & $-0.350^{\star \star}$ & -0.168 & 0.208 & -0.021 \\
\hline FEV1 [\%] & 0.168 & 0.105 & -0.161 & $-0.394^{* *}$ & $-0.287^{*}$ & -0.083 & 0.219 & 0.022 \\
\hline FEV1/FVC & 0.064 & 0.085 & -0.186 & $-0.428^{* *}$ & $-0.275^{\star}$ & -0.254 & 0.049 & -0.141 \\
\hline \multicolumn{9}{|c|}{ Maximal respiratory pressures } \\
\hline MIP [\%] & -0.097 & -0.125 & -0.239 & -0.118 & $-0.297^{*}$ & -0.221 & 0.134 & 0.045 \\
\hline $\operatorname{MEP}[\%]$ & $-0.338^{*}$ & $-0.382^{* *}$ & -0.136 & -0.039 & -0.096 & -0.158 & 0.003 & 0.108 \\
\hline \multicolumn{9}{|c|}{ Inflammatory biomarkers } \\
\hline Fibrinogen & -0.160 & -0.195 & $-0.379 * *$ & -0.209 & -0.163 & -0.194 & 0.050 & -0.107 \\
\hline CRP & 0.107 & 0.025 & -0.044 & -0.052 & 0.013 & 0.003 & 0.241 & 0.224 \\
\hline \multicolumn{9}{|c|}{ Physical activity capacity } \\
\hline $6 \mathrm{MWT}[\mathrm{m}]$ & 0.088 & 0.184 & $-0.339 *$ & $-0.581^{\star \star \star}$ & $-0.631^{* *}$ & $-0.558 * *$ & 0.170 & -0.155 \\
\hline \multicolumn{9}{|l|}{ Tests } \\
\hline HADs-d & 0.012 & -0.114 & 0.231 & $0.412^{* *}$ & $0.329 *$ & $0.409 * *$ & $0.277^{* *}$ & $0.387^{* *}$ \\
\hline HADs-a & 0.157 & 0.045 & $0.263^{*}$ & $0.400^{\star \star \star}$ & $0.380^{\text {** }}$ & $0.495^{* \star}$ & $0.266^{\star * *}$ & $0.359 * *$ \\
\hline MOCA[pts] & 0.033 & 0.076 & -0.080 & -0.208 & -0.125 & -0.108 & -0.201 & -0.254 * \\
\hline
\end{tabular}

*Correlation is significant at the 0.05 level (2-tailed); ** Correlation is significant at the 0.01 level (2-tailed) Abbreviations: FVC - Forced Vital Capacity; FEV1 - Forced Expiratory Volume in the first second; MIP -Maximum Inspiratory Pressure; MEP -Maximum Expiratory Pressure; HADs - Hospital Anxiety and Depression Scale; MoCA - Montreal Cognitive Assessment; 6MWD - 6 Minutes Walking Distance; CRP -C-Reactive Protein;

CAT - COPD Assessment Test; q1 -item cough; q2 -item phlegm; 93 -item chest tightness; $q 4$-item walking up hills and stairs; q5 -item doing activities at home; q6 - item confidence leaving my home; 97 - item sleep; 88 - item energy.

Maximal respiratory pressures were poorly correlated with CAT score, namely, MIP and CAT score $(r=-0.240, p$ $=0.075)$, and MEP and CAT score $(r=-0.174, p=0.200)$. On the contrary, MIP was significantly correlated with CAT item doing activities at home $(r=-0.297, p<0.05)$ than MEP which was significantly correlated with items cough $(r=-0.338, p<0.05)$ and phlegm $(r=-0.382, p<0.01)$. Fibrinogen, as an inflammatory biomarker, was poorly correlated with the total CAT score $(r=0.286, p=0.033)$, but it was significantly correlated with CAT item chest tightness ( $r=-0.379, p<0.01$ ) (fig. 1). In addition, MoCA score, poorly correlated with the CAT score $(r=-0.174, p=$ $0.200)$, revealed a stronger and significant correlation with individual item energy $(r=-0.254, p<0.05)$ (fig. 1).
Recent studies have proved the sustainability of the CAT questionnaire presenting it as a reliable instrument that can measure the COPD patient's quality of life compared to other tools usually used in this disease [20]. The main objective of the study was to analyse if individual CAT items are better correlated with different tests performed in COPD patients. Exacerbation in the last three months, anxiety and depression, decreased physical capacity and poorer lung function were all found to be significantly correlated with the total CAT score. Some studies have demonstrated that CAT score correlates with exacerbation severity and its impact on the health status [20]. We found COPD patients with higher CAT total score had exacerbation in the last three months, results consistent 


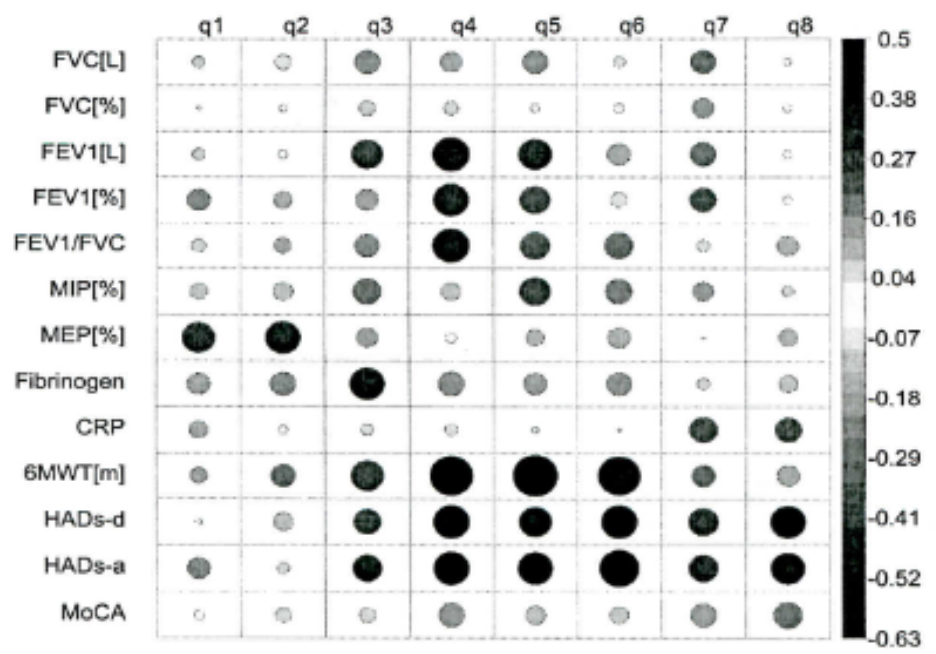

Fig. 1. Correlation coefficients between items of CAT and measured parameters Abbreviations: FVC - Forced Vital Capacity; FEV1 - Forced Expiratory Volume in the first second; MIP - Maximum Inspiratory Pressure; MEP - Maximum Expiratory Pressure; CRP - CReactive Protein; 6MWD - 6 Minutes Walking Distance; HADs - Hospital Anxiety and Depression Scale; MoCA - Montreal Cognitive Assessment;.

with those of Sang-Do Lee et al, who evaluated the relationship between the higher CAT score and shorter time to the first exacerbation or risk of exacerbations [21]. Different studies have shown that anxiety and depression are co-morbidities of COPD independently associated with an increased risk of exacerbation even in stable COPD forms. Burgel et al. found depression as the most important contributor to the Saint Georges Respiratory Questionnaire total score in patients with COPD [22]. Masaki Miyazaki et al. observed depression has a more important impact on increasing the CAT score than anxiety thus highlighting the psychiatric co-morbidities of COPD [23]. Consistent with these findings, we observed that HAD depression score was significantly correlated with several CAT items as walking up hills and stairs, doing activities at home, confidence leaving home and energycompare to the score of HAD anxiety, which was significantly correlated only with the total CAT score. Consistent with Young Seok Lee et al., who found that depression was associated better with the chest tightness, confidence leaving home, activity limitation at home, energy and sleep [24], we observed a significant association and presented good discrimination power of energy item. Thus, energy item can be a better indicator of depression compared to other COPD symptoms such as phlegm and cough in patients with COPD. The most important symptoms that limit the daily living activities among COPD patients, reducing gradually the functional status, are fatigue and dyspnoea [25]. One of the highest predictors of mortality in these patients is the level of physical activity and a decline in functional status can have a worse impact on the health status of the patients [26]. We noticed a drastic decrease of the functional capacity at higher CAT scores. Differences between medium, high and very high CAT score levels were statistically significant. The most significant correlation between CAT item and 6MWD was limitation doing activities at home. Carlos $\mathrm{H}$. Martinez et al. found similar results reporting that patients who had an increased CAT score and a reduced quality of life presented a shorter walking distance [27]. Interestingly, compared to our study, a link between dyspnoea and reduced physical capacity was described related to the first four CAT questions. Similar to our results, Martinez found significant correlation between higher CAT score and depression, leading to reduced physical activity, fatigue and poor sleep [27]. CAT score is well correlated with the functional status when it is evaluated with the help of the 6MWD test [28]. We found the same correlation between lower CAT score and increased functional status. Reduced physical activity can explain the significant correlation between MIP and CAT item doing activities at home. It is well known that the weakness of the respiratory musculature with reduced muscular resistance and strength can have important clinical consequences for patients with COPD, explaining partially common symptoms such as effort dyspnoea and reduced tolerance to physical exercise [29]. Patients with COPD comprise multiple comorbidities, including cognitive impairment and a greater risk of developing cognitive impairment than patients without COPD [30,31]. MOCA questionnaire is capable to detect minor cognitive impairment found that all patients with severe COPD suffered of minor cognitive impairment regardless of hypoxemia [31]. We also found cognitive impairment in COPD patients (MoCA score less than 26 points) without significant correlation between this test and total score of CAT, the only significant correlation being noticed for energy item. Relationship between systemic inflammation and CAT score is not very well studied with inconclusive and contradictory data about the correlation between CRP and CAT [32-34]. Compared to these studies, in our study CRP is inversely correlated with total score of CAT and the only significant correlation is between fibrinogen and CAT item chest tightness. One of the limitations of our study was that it had a small sample size although other studies that used COPD measurement instruments had also reduced number of patients.

\section{Conclusions}

This study demonstrated an independent association between different CAT items and other tools used in the assessment of patients with COPD. The most significant link was between CAT components related to walking up hills and stairs, limitations doing activities at home and confidence leaving home. The most powerful correlation was observed between these three questions and depression and 6MWD.

\section{References}

1.WELDAM, S.W.M., SCHUURMANS, M.J ., LIU, R., LAMMERS, J.W.J ., Int J Nurs Stud., 50, 2013, p. 688.

2.VESTBO, J., HURD, S.S., AGUSTI, A.G., et al. Am. J. Respir. Crit. Care Med., 187, nr 4, 2013, p. 347.

3.TUDORACHE, E., FILDAN, A.P., FRANDES, M., DANTES, E., TOFOLEAN, D.E., Clin Interv Aging. 12, 2017, p. 1281.

4.OTELEA, M.R., ARGHIR, O.C., ZUGRAVU, C., NAGHI, E., ANTONIU, S., RASCU, A. Rev. Chim. (Bucharest), 69, no. 2, 2018, p. 346.

5.GREULICH, T., AVERYANOV, A., BORSA, L., et al. Clin Respir J., 11, nr 1, 2017, p. 90. 
6.TIMAR, B., TIMAR, R., GAITA, L., et al. PLoS One, 11, nr 4, 2016, e0154654.

7.J ONES, P.W., HARDING, G., BERRY, P., et al. Eur Respir J., 34, nr 3, 2009, p. 648.

8.GUPTA, N., PINTO, L.M., MOROGAN, A., BOURBEAU, J. Eur Respir J., 44, nr 4, 2014, p. 873.

9.WEDZICHA, J.A., BRILL, S.E., ALLINSON, J.P., et al. BMC Med., 11, 2013, p. 181.

10.MIHAILOV, C., JIMBOREAN, G., RASCU, A., ARGHIR, O.C. J . Environ. Prot. Ecol., 2016, 17, nr 4, 2016, p. 1523.

11.***Material Biocompatibility and Standard for Plastic Manufacturing Systems/Components. United States Pharmacopeia (USP), 2016

12.***Medical Device Design using Polymer Compounds. https:// www.mexichemspecialtycompounds.com/blog/medical-devicedesign-using-polymer-compounds/3410/ Accesed on Jan, 2019

13.ANJA, W.K., AXEL, K., MURIEL, M.G., et al. Nutrition Journal, 10, 2011, p. 35.

14.NASREDDINE, Z.S., PHILLIPS, N.A., BÉDIRIAN, V., et al. J ournal of the American Geriatrics Society, 53, nr. 4, 2005, p. 695.

15.WASSERMAN, K., HANSEN, J.E., SUE, D.Y., et al. Principles of exercise testing and interpretation, 3rd edition. Philadelphia: Lippincott, Williams \& Wilkins; 1999.

16.*** CAT DEVELOPMENT STEERING GROUP. COPD assessment test health care professional user guide (accessed 12 nov 2017).

17.ZIGMOND, A.S., SNAITH, R.P., Acta Psychiatr Scand, 67, 1983, p. 361. 18.RASCU, A., POPA, D.E., ARGHIR, O.C., OTELEA, M.R. FARMACIA, 64, nr 6, 2016, p.819.

19.0TELEA, M., RASCU, A., POSTOLACHE, P. Rev.Med.Chir. Soc.Med.Nat. Iasi, 120, nr. 3, 2016, p.497.
20.J ONES, P.W., HARDING, G., WIKLUND, I., et al. Chest, 142, 2012, p.134e40.

21.SANG-DO, L., MING-SHYAN, H., J IAN, K., etal. Respiratory Medicine, 108,2014, p. 600.

22.BURGEL, P.R., ESCAMILLA, R., PEREZ, T., CARRE, et al. Respir Med, 107, 2013, p. 233.

23.MASAKI, M., HIDETOSHI, N., SHOTARO, C., et al. Respiratory Research, 15, nr. 1, 2014, p. 13.

24YOUNG, S.L., SUNGHOON, P., YEON-MOK, O., et al. J Korean Med Sci, 28, 2013, p. 1048.

25.DE TORRES, J.P., MARIN, J.M., MARTINEZ-GONZALEZ, C., et al. Chest, 146, nr. 1, 2014, p. 111.

26.TUDORACHE, V., OANCEA, C., AVRAM, C., et al. Wien Klin Wochenschr, 126, nr.1-2, 2014, p. 30.

27.MARTINEZ C.H., MURRAY, S., BARR, G.R., et al. Ann Am Thorac Soc., 14, nr. 5, 2017, p. 636.

28.GULART, A.A., MUNARI, A.B., QUEIROZ, A.P., et al. Chron Respir Dis., 14, $\mathrm{nr}$ 1, 2017, p. 37.

29.0ANCEA, C., FIRA-MLADINESCU, O., TIMAR, B., et al. Wien Klin Wochenschr, 127, nr. 9-10, 2015, p. 388.

30.HUNG, W.W., WISNIVESKY, J.P., SIU, A.L., ROSS, J.S. Am J Respir Crit Care Med., 180, nr. 2, 2009, p. 134

31.CRISAN, A., OANCEA, C., TIMAR, B., et al. Plos ONE, 9, nr. 7, 2014, p. e102468

32.TUDORACHE, E., OANCEA, C., AVRAM, C., etal. IntJ Chron Obstruct Pulmon Dis., 10, 2015, p. 1847.

33.SARIOGLU, N., HISMIOGULLARI, A.A., BILEN, C., et at. Rev Port Pneumol., 22, nr. 1, 2016, p. 11.

34.HYUNG, K.K., KANG, K., HYUN, L., et al. Int J ournal of COPD, 11, 2016, p. 3137.

Manuscript received: 27.03 .2019 